\title{
Study on the Color Characteristics of Dalian City
}

\author{
Ming Qi, Xu Lu and Quanhua Qian \\ Shenyang Jianzhu University, China
}

\begin{abstract}
This study is based on GB/T15608-1995 "The Chinese color system" and "Chinese architectural color card" as basic research tools, mainly using quantitative analysis (color digital processing), and qualitative methods(color map generalization) as auxiliary. We do the research regarding the urban construction of Dalian, and build the foundation for the scientific planning and management of urban color management. It was found that the color of Dalian has the characteristics of being concentrated in the areas of low and medium color and high brightness, and the contrast of cold and warm is sharp, where the warm tone is stronger than the cold tone, and the control of modern artificial facade material color is put forward. For the buildings with special volume or special function, the city color plan should include special control.
\end{abstract}

Keywords: Urban Color; Current Situation Investigation; Quantitative Analysis; Dalian City

\section{Urban Color Theory}

Urban color, also known as "urban environmental color", refers to the sum of the color features of the public space. To put it bluntly, the overall color relationship formed when the elements of urban composition, such as architectural color, road color, public facilities color, bridge color, green color and so on, are combined together, which can be called as urban color. Urban color not only includes natural factors, but also includes a large number of artificial environment, which is a reflection of urban civilization in the space environment. It relates to the quality of human habitat and reflects the connotation and quality of a city. By Albert Alberty, the architectural theorist, in 1452 "Ten books of architecture", the construction method of building facade color is recorded[1].The architect Le Corbusier stressed the importance of the use of the facade color language in architectural design, and systematically expounded his understanding and application principles of color through engineering practice and papers.[2].People who also paying attention to the study of architectural color are the architect Walter Gropius.[3], Van Doesburg[4] and Bruno Taut[5]Wait. In 1960s, the most prominent research in the field of color science was the color geography theory from the French colorist Jean-Philippe Lancelot. The theory of color geography emphasizes that the local humanistic environment and natural environment have decisive significance to the color, and advocates that the color environment should be integrated and repaired through the investigation and analysis of the color environment.[6]. In the field color sampling, Japanese Color Planning Center, which is also the most important research institution for urban environment color in Asia, used the electronic color spectrometer to systematically analyze the urban color environment, which includes the comprehensive natural geographical factors and cultural geographical factors, to provide a more accurate color database for urban color research.

In recent years, Chinese scholars have carried out continuous research in the field of urban color. Yin Sichen believes that urban color has environmental, relatively permanent, public and cultural features.[7].In author's opinion, among many characteristics of urban color, there are two kinds that are directly related to the generation mechanism of urban color. One is the randomness of the individual and the fragmentation of the whole. From the current situation, the choice of urban color is mostly based on the individual preference of the builder, and has a strong contingency. The urban color of a certain area, as a sum of many occasional cases, is 
influenced by the social, cultural, economic, climatic and other factors of the region during its formative period. However, it is difficult to say how one factor plays a role in it.and whether its impact is decisive. The other is the permanence of the individual and the inheritance of the whole. Permanence refers to the fact that, excepting signs and advertising colors, other building facades, bridges and urban sketches are relatively existing in a long term, and natural colors such as sky, river, soil and so on are almost eternal. Therefore, for a built city area, the basic color environment has a relatively permanent feature, which generally will not rapid, frequent change. Therefore, at any point of time, a built area of environmental color has a historical heritage, its planning and guidance must be based on the respect for the current situation. In this paper, the current situation of urban color in Dalian City of China is analyzed in detail by using the method of Color Geography. Based on this, the problems of urban color are summarized, and suggestions for improvement are put forward.

\section{Research and Design}

\subsection{Scope of Research}

Because of its unique natural conditions and long history of culture, Dalian has formed a unique urban color.. Therefore, the two aspects long-term investigation of the natural color and architectural color deeply into Dalian scenic spots and urban areas, has an in-depth understanding and analysis of the causes and characteristics of Dalian city color. We mainly investigates four areas: Dalian, Lvshun, Jinzhou and Jinzhou Development Zone, and divides Dalian into four sub-parts: Ganjingzi District, Zhongshan District, Shahekou District and Xigang District. Among them, the natural color of the city is mainly aimed at the color content of the Xinghai Bay, Laohu Beach, Golden Beach, Forest Zoo and Binhai Road, as well as the natural geography within its scope. The humanistic color of the city is mainly aimed at the subjective color content of various scenic spots and cultural and historical buildings in Dalian as well as the culture. The current color of the city is mainly aimed at the existing buildings in Dalian and the existing artificial landscape and the color content of the construction.

\subsection{Research Methods}

The color information of the building surface is divided into two groups, one is the main color of the building, that is, the outer wall of the building and the surface color of the roof; Another group is the embellishments, from doors and windows, window panels, frames and wall bases, and so on. Later color surveys largely followed this method of dividing the building by standard elements. However, the contemporary architecture in the design concept and materials have undergone great changes, making the traditional division method is no longer applicable. Judging from the existing cases of color investigation in China, the binary classification method of "main color-embellishment color" has still been generally adopted, but sometimes the concept of "auxiliary color" is added, and sometimes it means the color that occupies a secondary position in some main colors. Sometimes it refers to the embellishment.In this study, from an analytical point of view, the binary classification of "main color-embellishment color" is adopted, but there is more than one color in each layer. In terms of the number of main colors, The architecture is divided into "mono-color", "bi-color" and even "tri-color".

Through the current sampling, the original information of urban color is obtained, and the original information is converted into the standard data of hue, brightness and saturation through the basic principle of color, and the quantitative analysis is carried out. Based on the background of city development and the degree of color preference obtained by questionnaires, the author makes a comprehensive analysis, obtaining the basic judgment on the color of Dalian city, and finds out the direction of improvement and adjustment, which provides references for urban color planning.

\subsection{Research Tools}

This study uses the Chinese color system to describe and analyze urban color.The Chinese color system is established on the basis of synthesizing the advantages and disadvantages of all kinds of color systems in the world and it is on the basis of the Chinese visual experiments. It describes and calibrates the color by the three 
attributes: color hue, brightness and saturation.In this study, mainly using GB/T15608-1995 "The Chinese color system", based on the "Chinese Building color card"to investigate and record the color of urban buildings, and "Chinese architectural color card"Electronic software is used to preprocess the color data from the survey.
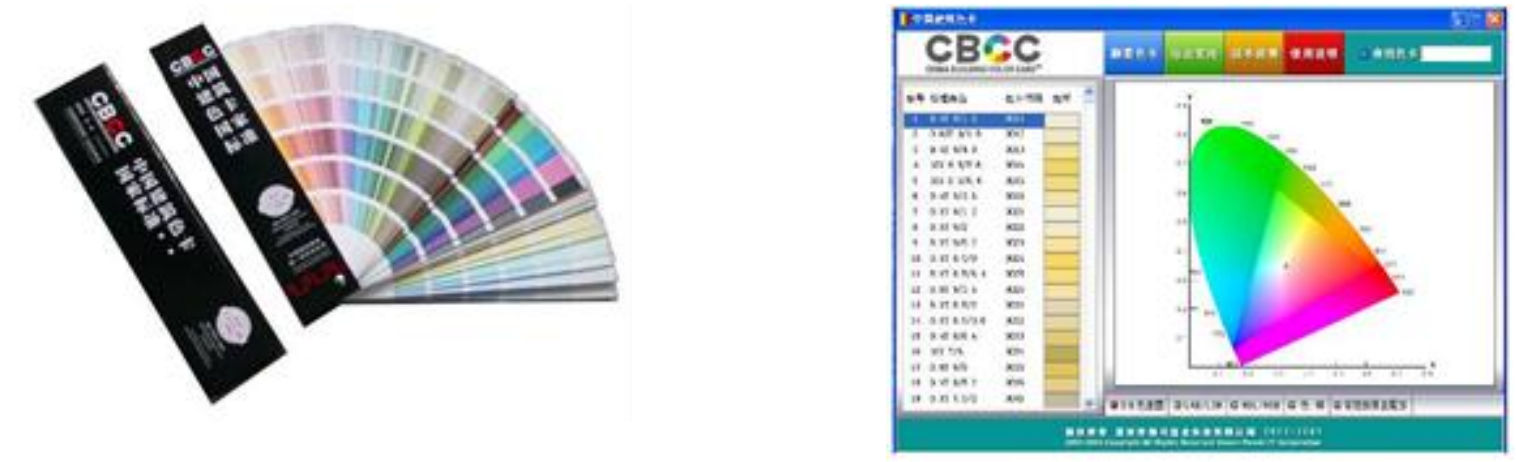

Fig. 1. Chinese Building Color Card and software

\section{Urban Background Color}

\subsection{Natural Colors}

Dalian is located in the warm temperate zone of the Northern Hemisphere, with oceanic characteristics of the warm temperate continental monsoon climate, is the warmest place in the Northeast, where winter without cold, summer without heat, and four seasons are distinct. Such climate nurtures nourish the growth of natural plants in Dalian, and it is unique among whole northeast regions in China. It is more grand compared with southern climates in China, with its bright and different colors, forming a unique Dalian.

Dalian has many mountainous hills, less lowland plain, with karst topography and marine erosions everywhere. Dalian also has reef coastline, beach coastline and mixed coastline which also makes the color of its soil and rocks unique. The soil in Dalian is sandy and gravel soil, and a large number of pebble, gravel, gravel and other materials are used to pave the ground, so the overall tone is yellowish, and has more variety than the inland areas.

Dalian is located in the mid-latitude area, the climate is mild, suitable for plant growth, so that it can give birth to a large number of color species. The main characteristics of vegetation color is the large amount of green trees and mixed growth of shrubs, forming a unique green mountain, and become the main background of the city. However, because of latitude, the flowering plants in Dalian are relatively scarce, the vegetation color is relatively simple, and its change is significant in autumn and winter. . 


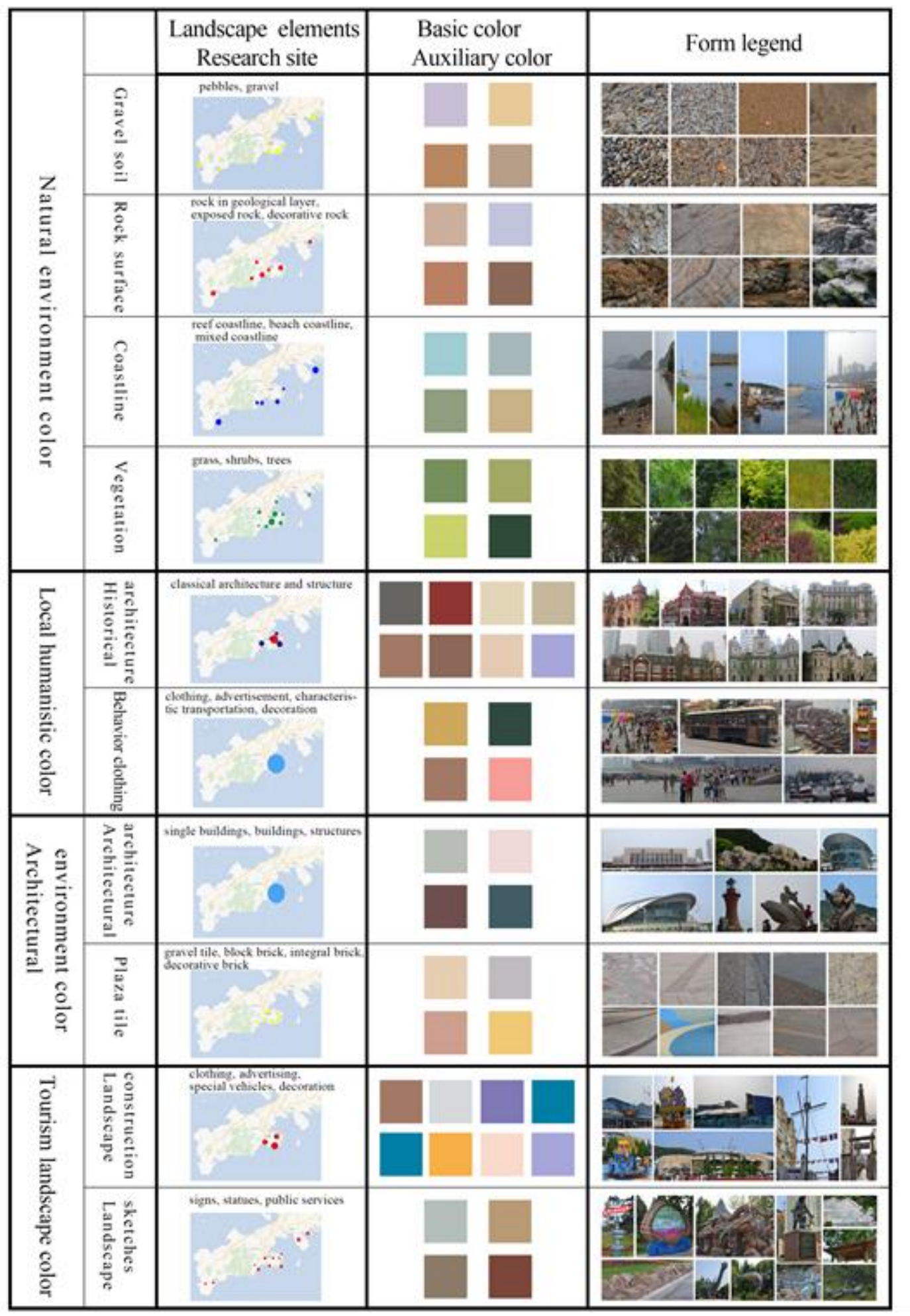

Fig. 2.Classification analysis of urban color in Dalian

\subsection{Local Humanities}

Dalian is a short-history city, with only small towns and a small population before 1880s. After modern times, Dalian was successively occupied by the tsar Russia and Japan. The colonial activities had a great influence on the constitution of the city's humanistic color. In 1897, during the period of Russian construction, Dalian was comprehensively planned and constructed, which made the color of Russian architecture and park prominent. Today, Dalian can still see the Eastern Orthodox Church and buildings with Russian style. During 
the period of Japanese occupation, Dalian's urban planning scope was expanded, and a concentrated Japanese residential area was formed. The style of urban architecture color was more elegant and chic than that of Russian occupation period. At the same time, as a coastal city, Dalian is also influenced by the blending of the regional culture around the Bohai Sea and the culture of various nationalities in the Northeast, as well as the influence of the culture of $\mathrm{Ma} \mathrm{Zu}$ and other coastal cultures.

After the founding of New China, Dalian became one of the most important coastal industrial cities in China. After entering the period of reforming and opening up, in order to develop urban tourism and improve urban landscape to attract investments, Dalian has built a large number of image squares and centralized green spaces, highlighting the color of plaza paving and focused on the city along the coastline of the landscape corridor. The urban artificial color then became more and more rich.

\section{Urban Building Color}

\subsection{Color Features}

From the overall view, the distribution of the main color samples in Dalian is not balanced, showing a very obvious trend towards the three regions. In the area of low and medium color, high brightness concentration. As you can see in figure 1 and the table, the bright $(\mathrm{V}>5)$ sample accounted for $92 \%$, low saturation $(\mathrm{C}<$ 7)samples accounted for $72 \%$, while the samples with both these two features accounted for $70 \%$. This concentrated distribution is related to the characteristics of the architectural color itself, and the light tone is easy to achieve a harmonious and consistent effect with the outdoor color environment.

There is a tendency to concentrate in two directions: cold and warm. In addition to the high brightness and low color concentration, the color samples also show the trend of concentrating in the cold and warm directions. The concentrated samples uses two axis of 5YR and 2.5PB color as the core, and they are distributed in two sectors on the color ring. As can be seen from figure 2, there are more samples of warm tone and a larger range of sectors is shown $(5 \mathrm{R} \sim 7.5 \mathrm{Y})$. The distribution of cold colors are more concentrated and the range of sectors is smaller (10 PB 5B). At all stages of color, the samples in sectors of (5 R 10 PB) and (10Y $\sim 5 \mathrm{~B})$ are rare.

Warm tone is stronger than cold tone. In areas of $(\mathrm{C}<6)$, samples in cold tone $(5 \mathrm{P} \sim 7.5 \mathrm{~GB})$ are fewer samples in warm tones $(2.5 \mathrm{RP} \sim 5 \mathrm{Y})$; At areas of $(\mathrm{C}>6)$, cold tone has sample, which however are also less than that in warm tone. On the whole view, the number of warm tone samples is about 2 times that of cold tone, which is dominating, but the difference between two quantities is still not obvious.

From the overall view, the results of the three regional clusters of colors are as follows: The characteristics of urban color is not obvious, the collocation between cold tone and warm tone may form a contrast and harmony in some parts, but it gives a confused and uncoordinated psychological feeling on the whole. From the point of view of planning, it should be determined that the tone of the future color development of Dalian is towards a higher brightness, lower color and warmer tone.
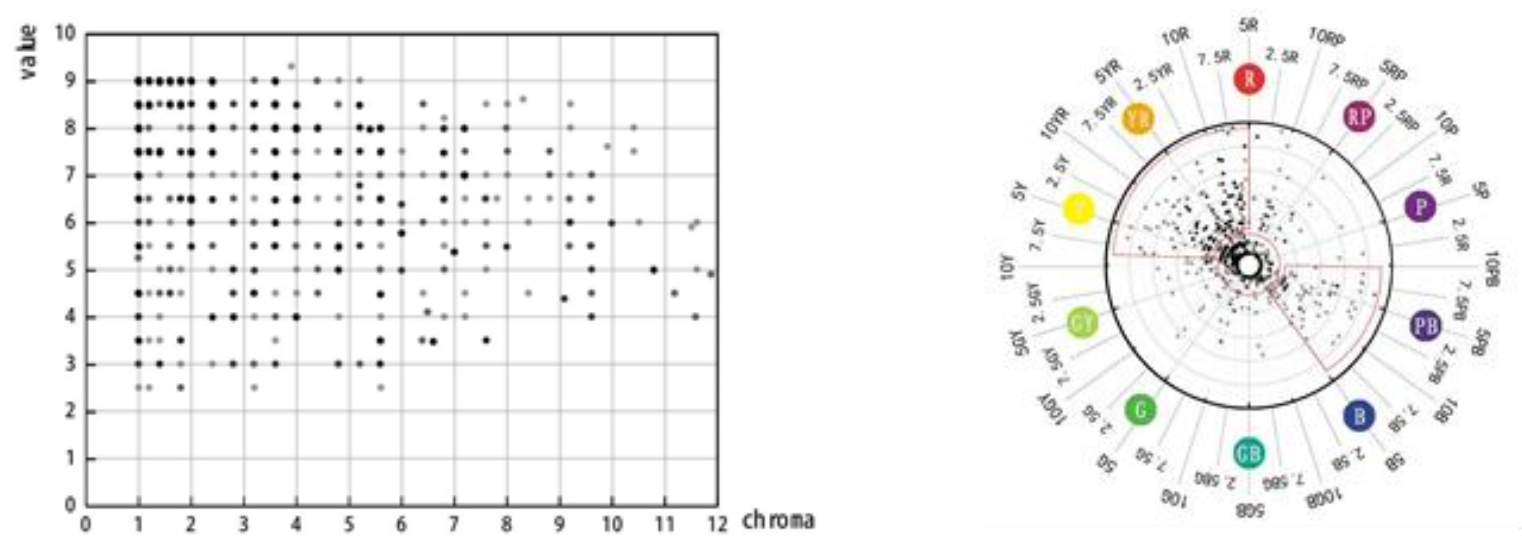

Fig. 3.Scatter diagram of dominant color in architecture facade 


\subsection{Main Issues}

The tone of city color is high brightness, low color, and trends to a warm tone, which is in harmony with the natural environment of the northern coastal city in China, and reflects the historical context of Dalian, which is a very successful landmark landscape with overall color characteristics. However, there are many color landscape problems in the study area at the same time. First of all, high saturation, warm color samples, with the obvious contrast and mixed distribution of cold, warm and gray colors results in the overall feeling of confusion. Secondly, the results show that glass and paint are the most widely used building materials, easy changeable paint colors are most likely to lead to color effect out of control. Third, commercial and official buildings in the city center district has its iconic features. Its color tendency of low color, cold tone is the dominant factor to form the color tone of Dalian. Fourth, the color tendency of residential, commercial and tourist categories is obviously different from that of office. Colors in some sections is quite different from the overall color planning.

\subsection{Recommendations for Improvement}

The concept of "dominant color" or "dominant color system" is used in urban planning. Different backgrounds, different functional locations of the region will inevitably have a completely different color tendency. In the process of architectural design, it is necessary to comprehensively consider the functional orientations of the area of architecture for the whole city, so that the colors of the urban space can reflect the natural and humanistic characteristics of the region, and present unique color characters at the same time.

The historical and cultural district of Dalian and the residential district with local characteristics should follow the original traditional color of the city. On the basis of analyzing and summing up the color characteristics of each historical building area, the color control area or the style and feature coordinated area are delimited within a certain range around the corresponding block, and the different degree of color control is carried out in each district. These actions are in order to protect the historical features of the city, and continue having representative traditional colors. For the residential areas with local characteristics, the colors of the residential buildings should try to form a echo with the traditional colors.

Under the premise of ensuring the harmonious overall image of the regions, for the new commercial district and development zone of Dalian city, the color control scope of the building should be looser, aiming at the different color guidance of the building with different functions and volumes. Giving modern feelings from the whole, but in the small volume buildings or the detailed structure of the buildings, we should manifest the Dalian historical characteristics.

In the case of building monomer in each region, we should avoid "visual shock" as the only way of visual communication. The color of the building monomer should meet the requirements of the regional environment to avoid abrupt in a single building color, which will affect the overall image of the region.

For individual buildings that affect the overall effect of urban environment, facade rectification and painting can be carried out; For buildings that affect the overall view of urban environment and are not easy to be repainted, street greening can be used, such as planting trees that are closely around the buildings and isolating them visually, in order to reduce their impact on the overall effect of urban environment.

To sum up, urban color planning should take the natural conditions and humanistic characteristics into account, and comprehensively consider the functional orientation of construction sites in the city, and from the regional point of view, providing references and suggestions for the selection of architectural colors, in order to achieve harmonious and beautiful integration with the whole city.

\section{Acknowledgement}

This article is supported by National Natural Science Foundation of China 'Study on the Urban Color Characteristics and its Preservation Methods of Cold Cities in Northeast China Based on Color Geography'(51778375) . 


\section{References}

[1] Alberti LB. De re aedificatoria; 1452. English translation by Leoni G(1755), reprinted as The ten books of architecture. New York: Dover;1986.

[2] Ozenfant A, Le Corbusier. Apre's le Cubisme. Paris: Edition des Commentaires; 1918. Spanish translation, Despue's del Cubismo. In: Pizza A, editor. Acerca del Purismo. Escritos 1918-1926. Madrid: El Croquis Editorial; 1994.,pp.847.

[3] Gropius W. Scope of total architecture. New York: Harper \& Row;1955.

[4] José Luis Caivano. Research on Color in Architecture and Environmental Design: Brief History, Current Developments, and Possible Future.Color research and application.2006(4).pp.350-363. https://doi.org/10.1002/col.20224

[5] Taut B. Lecture on the renaissance of color published in Farbe am Hause, 1st German Colorist Congress. Hamburg: Bauweltverlag;1925. Shorter version translated into Spanish, in: Düttmann M,Schmuck F, Uhl J, editors. El color en la arquitectura. Barcelona:Gustavo Gili; 1982,pp.12-15.

[6] Lenclos J. P., Lenclos D. Colors of the world, W. W. Norton and \& Company Inc., New York, 2004.

[7] Yin Si-jin. Urban Color Landscape Planning and Design[M]Nanjing:Southeast University Press, China.2004,pp.13-14. 